\title{
The effect of nocturnal blue light exposure from light-emitting diodes on wakefulness and energy metabolism the following morning
}

\author{
Momoko Kayaba • Kaito Iwayama • \\ Hitomi Ogata $\cdot$ Yumi Seya $\cdot$ Ken Kiyono • \\ Makoto Satoh $\cdot$ Kumpei Tokuyama
}

Received: 10 July 2014/ Accepted: 21 July 2014/Published online: 5 August 2014

(C) The Japanese Society for Hygiene 2014

\begin{abstract}
Objectives The control of sleep/wakefulness is associated with the regulation of energy metabolism. The present experiment was designed to assess the effect of nocturnal blue light exposure on the control of sleep/wakefulness and energy metabolism until next noon.

Methods In a balanced cross-over design, nine young male subjects sitting in a room-size metabolic chamber were exposed either to blue LEDs or to no light for $2 \mathrm{~h}$ in the evening. Wavelength of monochromatic LEDs was $465 \mathrm{~nm}$ and its intensity was $12.1 \mu \mathrm{W} / \mathrm{cm}^{2}$.

Results During sleep, sleep architecture and alpha and delta power of EEG were similar in the two experimental conditions. However, the following morning, when subjects were instructed to stay awake in a sitting position, duration judged as sleep at stages 1 and 2 was longer for subjects who received than for those who received no light exposure. Energy metabolism during sleep was not affected by evening blue light exposure, but the next morning energy expenditure, oxygen consumption, carbon dioxide production and the thermic effect of breakfast were
\end{abstract}

\section{Kayaba $\cdot$ K. Iwayama $\cdot$ Y. Seya}

Graduate School of Comprehensive Human Sciences, University

of Tsukuba, Tsukuba, Japan

H. Ogata $\cdot$ K. Tokuyama $(\bowtie)$

Faculty of Health and Sports Sciences, University of Tsukuba,

Tsukuba, Japan

e-mail: tokuyama@taiiku.tsukuba.ac.jp

K. Kiyono

Graduate School of Engineering Science, Osaka University,

Osaka, Japan

M. Satoh

Faculty of Medicine, University of Tsukuba, Tsukuba, Japan significantly lower in subjects who received blue light exposure than in those who received no light exposure. Conclusions Exposure to low intensity blue light in the evening, which does not affect sleep architecture and energy metabolism during sleep, elicits drowsiness and suppression of energy metabolism the following morning.

Keywords Blue light · Light-emitting diodes (LEDs) · Thermic effect of food - Metabolic chamber - Sleep

\section{Introduction}

The artificial light environment has experienced a dramatic change during the last decade. The use of light-emitting diodes (LED)-based lighting has been increasing since the innovation of high-brightness blue LEDs in mid-1990s. Supported by energy policy in each country, it is predicted to replace incandescent bulbs and fluorescent lamps to provide an energy-efficient and long-lasting option for everyday use in many areas of the globe. LED-based white light consists of either blue LEDs with yellow phosphors or a combination of red, green and blue LEDs. Compared with incandescent bulbs, fluorescent lamps and natural light, the LED-based white light is extremely enriched with a blue light component [1]. The LEDs are also used for visual displays terminals (VDT), and VDTs are standard equipment both at work and at home in the front of computers, TVs, smart phones and games in industrialized societies.

It is known that nocturnal light exposure elicits a suppression of melatonin secretion $[2,3]$ and alerting response [4]. In 2001, short wavelength light (446-483 nm) was shown to be the most potent wavelength region to suppress melatonin secretion in humans, and this finding suggested 
the presence of a novel photoreceptor which is responsible for circadian photoreception and is distinct from rod and cone photoreceptors for vision $[5,6]$. Subsequent studies identified retinal ganglion cells, which contain a new photopigment named melanopsin, which is the most sensitive to short wavelength blue light [7-9].

Exposure to short wavelength light in the evening induces many psychological and physiological effects, including increased subjective alertness [10,11], heart rate and body temperature [11]. In these previous studies, the effects of short wavelength light were assessed during or immediately after light exposure. An exception is a study by Münch et al. [12], in which the impact of blue light exposure on sleep EEG and sleep architecture was studied. After evening exposure to blue light, EEG slow wave activity decreased slightly during the first and increased significantly during the third sleep cycles. Furthermore, core body temperature was elevated during light exposure and at the beginning of sleep, but the next morning, suggesting a circadian phase delay. The possibility that the effect of evening light exposure persists beyond the sleeping period remained to be evaluated.

The notion that sleep and energy metabolism are related is supported by several lines of evidence. Neuroactive substances that affect sleep often affect energy metabolism or feeding behavior as well, and examples of such dualacting neurosubstances are leptin, insulin, orexin, neuropeptide Y, etc. [13]. Epidemiological studies have identified short sleep duration as a risk factor for the development of obesity [14-17], and experimental intervention confirmed that sleep curtailment is associated with endocrine modifications such as decreased secretion of leptin and increased secretion of ghrelin [18-20]. Our previous study showed that energy expenditure and substrate oxidation during sleep were related to sleep stages [21]. Taken together, it is plausible that if exposure to short wavelength light in the evening affects sleep architecture, it might also affect energy metabolism during sleep.

This study was designed to assess the effect of nocturnal blue light exposure from LEDs on sleep and energy metabolism until next noon using polysomnography (PSG) and a room-size metabolic chamber, respectively. PSG, which measures EEG, chin EMG and eye movement, is the most popular method to evaluate sleep architecture, and it is used in clinical settings to diagnose sleep disorders and in sleep research to evaluate sleep quality. Usually, healthy people experience sleep cycles in 90-min intervals. Sleep consists of non-REM sleep and REM sleep. Non-REM sleep includes stage 1 and stage 2 (both light sleep) and slow wave sleep (deep sleep). Indirect calorimetry with a room-size metabolic chamber provides a controlled environment, in which energy metabolism can be continuously measured for a long period of time including sleep.

\section{Materials and methods}

Study subjects

Nine healthy young male volunteers participated in this study. The subjects' characteristics are presented in Table 1. Volunteers who were involved in night work, planned long-distance jet travel during the study period, had irregular sleeping schedules, had a smoking habit, and were taking any drugs known to affect sleep and metabolism were excluded from this study. Subjects had no complaints of sleep disorders (Pittsburgh sleep quality index $<5.5$ ). The day before the study, subjects were asked to keep a regular sleep-wake schedule, and to abstain from exercise and consumption of alcohol and caffeine. We verified the sleep-wake schedule and activity during daytime using a wrist motion sensor ActiGraph (Ambulatory Monitoring Inc., Ardsley, NY) and a self-reported diary.

Ethical approval for the study was granted by the University of Tsukuba Ethics Committee and was in compliance with the Declaration of Helsinki. All volunteers confirmed their compliance by written informed consent.

\section{Study protocol}

The study was carried out in a few weeks interval in a balanced cross-over design with intrasubject comparisons. In each session, the subjects were admitted to the laboratory $5.5 \mathrm{~h}$ before their habitual bedtime (Fig. 1). After preparation for polysomnographic sleep recordings, dinner $(842 \pm 123 \mathrm{kcal}$, mean $\pm \mathrm{SD})$ was served under dim light ( 2 lux). The subjects entered into the metabolic chamber and remained in a sitting upright position for $4 \mathrm{~h}$ until their habitual bedtime. After $2 \mathrm{~h}$ of dark adaptation (0 lux), subjects were exposed to blue light or remained in the dark for $2 \mathrm{~h}$. Wavelength of monochromatic LED (Panasonic, Osaka, Japan) was $465 \mathrm{~nm}$ and horizontal irradiance of the light at eye level was $7.02 \mu \mathrm{W} / \mathrm{cm}^{2}$. Subjects exposed to blue light in a vertical direction from the ceiling were instructed not to gaze into the light source. During the 4-h sitting period in the metabolic chamber, they were allowed to listen to music or radio. To further ensure wakefulness, a trained technician was constantly present in the adjacent room checking EEG traces on the screen for sleep signs. The subjects were contacted through an interphone at 15-min interval and every time when eyes were closed for several seconds. Epochs of sleep at stages 1 and 2 during scheduled wakefulness were observed but there were no significant differences in the sleep stages between the two conditions $(p=0.23$; Wilcoxon signed rank test). Following a 2-h period with or without blue light exposure, the subjects went to bed and slept for $8 \mathrm{~h}$. 


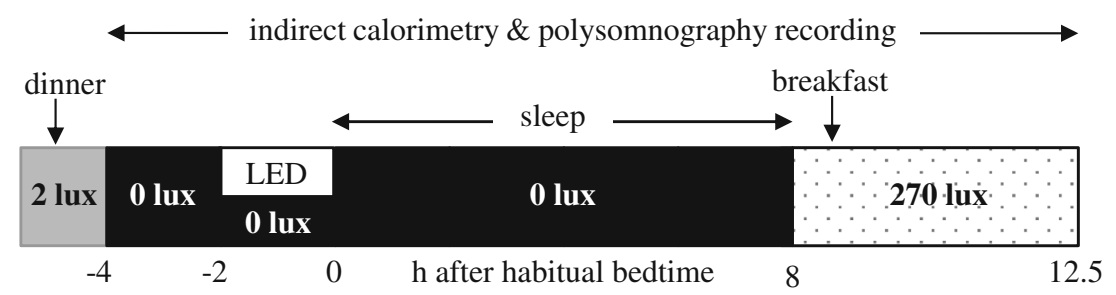

Fig. 1 Study protocol. The subjects were admitted to the laboratory $5.5 \mathrm{~h}$ before their habitual bedtime. Under dim light condition (2 lux), electrodes and sensors of polysomnographic recording were attached, and dinner was served $5 \mathrm{~h}$ before habitual bedtime. Subjects entered into the metabolic chamber and remained in a sitting upright position until bedtime. Following $2 \mathrm{~h}$ of dark adaptation ( 0 lux), subjects were

The next morning, the subjects stayed in the chamber under fluorescent light (270 lux) until noon. Basal metabolic rate was measured for $30 \mathrm{~min}$ in a supine position, followed by breakfast $(791 \pm 118 \mathrm{kcal})$. Subjects were instructed to remain seated and to stay awake until noon, and they were allowed to read and listen to music. However, when the subjects fell asleep in the morning hours, we did not intervene to wake them up.

The four meals prior to and during the experiment were individually standardized according to the estimated energy requirement for Japanese individuals [22], assuming physical activity level to be 1.4. Expressed as a percentage of total energy, the standardized meals contained $15.0 \pm 1.2 \%$ protein, $23.2 \pm 2.2 \%$ fat, and $60.7 \pm 1.8 \%$ carbohydrate (mean $\pm \mathrm{SD}$ ).

\section{Measurements}

\section{Sleep recording}

Sleep was recorded polysomnographically using Alice 5 (Philips Respironics, Tokyo, Japan). Four EEGs (C3/A2, C4/A1, O1/A2, and O2/A1), two electrooculograms, one submental electromyogram and one electrocardiogram were recorded. The records were coded, and 30-s epochs were used to score sleep stages according to standard criteria [23] by an investigator without knowledge of the conditions.

\section{Indirect calorimetry}

Energy metabolism was measured with a room-size metabolic chamber (FHC-15S, Fuji Medical Science Co., Ltd. Chiba, Japan). The airtight chamber measures $2.00 \times 3.45 \times$ $2.10 \mathrm{~m}$, having an internal volume of $14.49 \mathrm{~m}^{3}$. The chamber is furnished with an adjustable hospital bed, desk, chair, washbasin, and toilet. Air in the chamber was pumped out at a rate of $70 \mathrm{~L} / \mathrm{min}$. Temperature and relative humidity of incoming fresh air were controlled at $25.0 \pm 0.5{ }^{\circ} \mathrm{C}$ and $55.0 \pm 3.0 \%$, either exposed to blue light LEDs or remained in the no light condition for a subsequent $2 \mathrm{~h}$ period. After $8 \mathrm{~h}$ of sleep, the basal metabolic rate was measured for $30 \mathrm{~min}$ in a supine position, followed by breakfast, and the subjects stayed in the chamber under fluorescent light (270 lux) until noon

respectively. Concentrations of oxygen $\left(\mathrm{O}_{2}\right)$ and carbon dioxide $\left(\mathrm{CO}_{2}\right)$ in outgoing air were measured with high precision by online process mass spectrometry (VG Prima $\delta \mathrm{B}$; Thermo Electron Co., Winsford, UK). Precisions of the mass spectrometry, defined as the standard deviation for continuous measurement of calibration gas mixture $\left(\mathrm{O}_{2} 15 \%, \mathrm{CO}_{2} 5 \%\right)$, were 0.0016 and $0.0011 \%$ for $\mathrm{O}_{2}$ and $\mathrm{CO}_{2}$, respectively. At every $5 \mathrm{~min}, \mathrm{O}_{2}$ consumption and $\mathrm{CO}_{2}$ production rates were calculated using an algorithm for improved transient response [24].

\section{Thermometry and heart rate}

Temperature of a subject's external auditory canal was continuously recorded throughout the study, and data were collected every $30 \mathrm{~s}$ using a thermistor probe (ITP010-27, Nikkiso-YSI, Tokyo, Japan) and a data logger (N540, Nikkiso-YSI, Tokyo, Japan). Standard electrocardiogram leads were placed on the lateral thorax and on the sternum using electrocardiogram monitor (DS-2151, Fukuda-Denshi, Tokyo, Japan).

Data analyses

\section{Spectral analysis of the EEG}

The C3-A2 and O1-A2 EEG recordings were sampled at $200 \mathrm{~Hz}$ and analyzed using the techniques of the discrete fast Fourier transform. The fast Fourier transform was conducted on an EEG record length of $5 \mathrm{~s}$ to obtain a frequency resolution of $0.2 \mathrm{~Hz}$. Each $5 \mathrm{~s}$ segment of the EEG signal was first windowed with a Hanning tapering window prior to computing the power spectra. The power content (expressed as $\mu \mathrm{V}^{2}$ ) for each $30 \mathrm{~s}$ epoch of sleep was determined as the average power across the six $5 \mathrm{~s}$ segments of the EEG [25]. The spectral distribution was categorized into the following frequency bands: delta $(0.75-4.00 \mathrm{~Hz})$, theta $(4.10-8.00 \mathrm{~Hz})$, alpha $(8.10-12.00 \mathrm{~Hz})$, sigma $(12.10-14.00 \mathrm{~Hz})$ and beta $(14.10-30.00 \mathrm{~Hz})$. The power in each frequency bandwidth was expressed as a 
percentage of total power in each $30 \mathrm{~s}$ epoch of sleep. We used EEG from $\mathrm{C} 3$ to $\mathrm{A} 2$ for analyzing delta power and EEG from $\mathrm{O} 1$ to $\mathrm{A} 2$ for analyzing alpha power.

\section{Calculation of thermic effect of food}

The thermic effect of food was calculated as an accumulated increase in energy expenditure above the basal metabolic rate for $3 \mathrm{~h}$ after breakfast. The thermic effect of food was expressed as a percentage of the energy content of breakfast [26].

\section{Statistics}

Wilcoxon signed rank test was used to compare two conditions with the level of statistical significance at $5 \%$. Statistical analysis was performed using IBM SPSS Statistics 20 (version 20.0; SPSS Japan, Tokyo, Japan).

\section{Results}

\section{Sleep stages}

The visually scored sleep stages are summarized in Table 2 . There was no significant difference in sleep architecture during bedtime between the two experimental conditions. However, evening blue light exposure induced a significant increase in sleep at stages 1 and 2 the next morning. Slow wave sleep (stages 3 and 4) and REM sleep were not observed in the morning in a sitting position.

\section{EEG spectra}

The alpha and delta power of EEG were shown as percentage of total power (Fig. 2). During the 2-h light exposure period, alpha power in the blue light condition was significantly lower than that in the no light condition $(p<0.05)$. During sleep, alpha and delta power of EEG were similar between the two trials. The following morning, alpha power of EEG in the blue light condition was higher than that in the no light condition, but the difference was not statistically significant $(p=0.051)$.

\section{Energy metabolism}

There are no significant differences in energy metabolism during the dark adaptation, the light exposure and the sleep periods (Fig. 3). The following morning, energy expenditure $(p=0.050)$, oxygen consumption $(p<0.05)$ and carbon dioxide production $(p<0.05)$ were significantly
Fig. 2 Delta and alpha power of EEG as percentage of total power. Average $\pm \mathrm{SE}$ in 30-min intervals were plotted for blue light (open circle) and no light (filled circle) conditions. The percentage of alpha power in the blue light condition was significantly lower than that in the no light condition during the light exposure $(p<0.05$; Wilcoxon signed rank test)

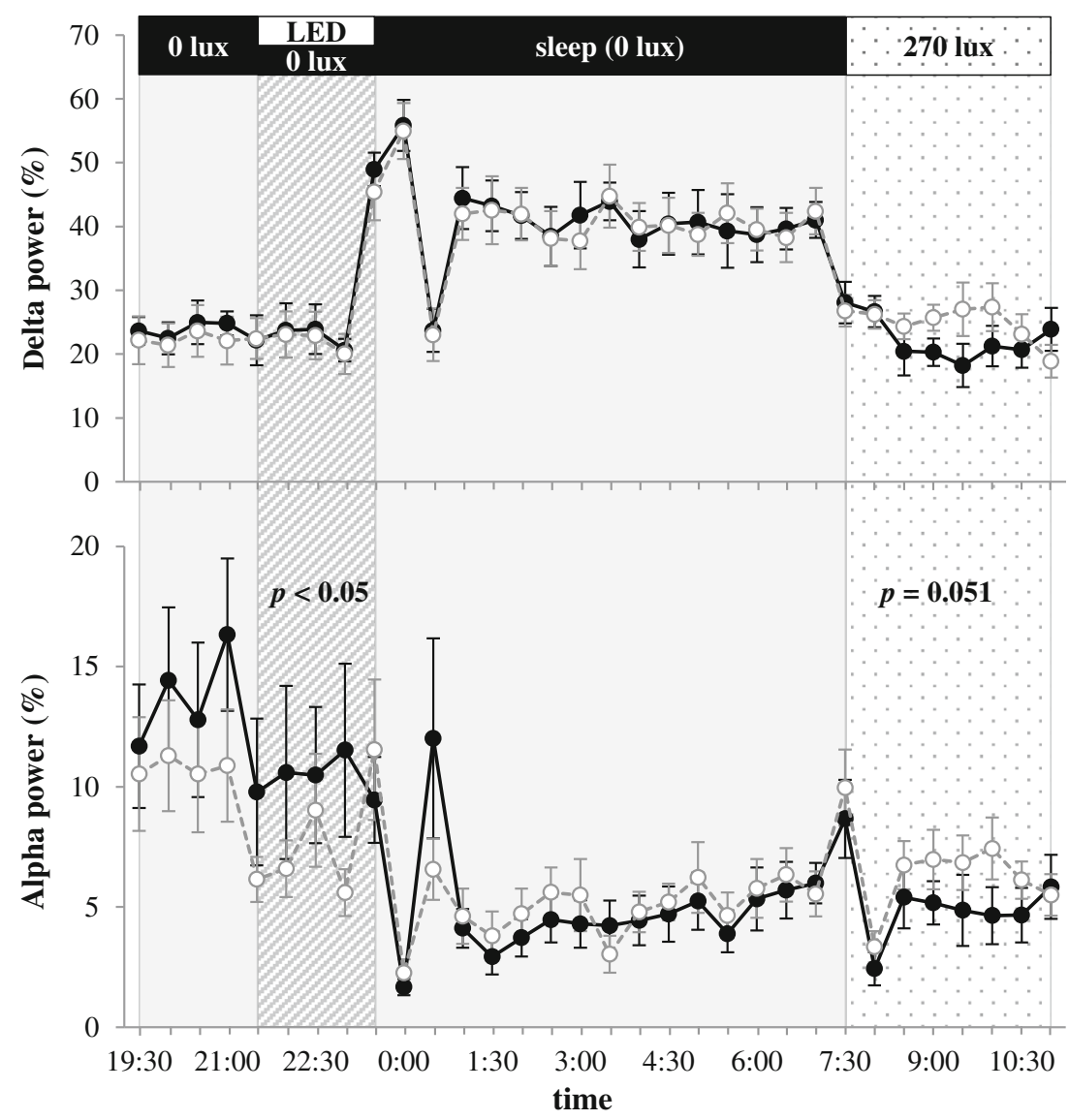


Fig. 3 Time course of energy metabolism. Average \pm SE of energy expenditure, oxygen consumption, carbon dioxide production and respiratory quotient in 30 min intervals were plotted for blue light (465 nm, open circle) and no light (0 lux, filled circle) conditions. The morning after evening blue light exposure, energy expenditure, oxygen consumption and carbon dioxide production were significantly lower than those not exposed to evening light ( $p \leq 0.05$; Wilcoxon signed rank test)

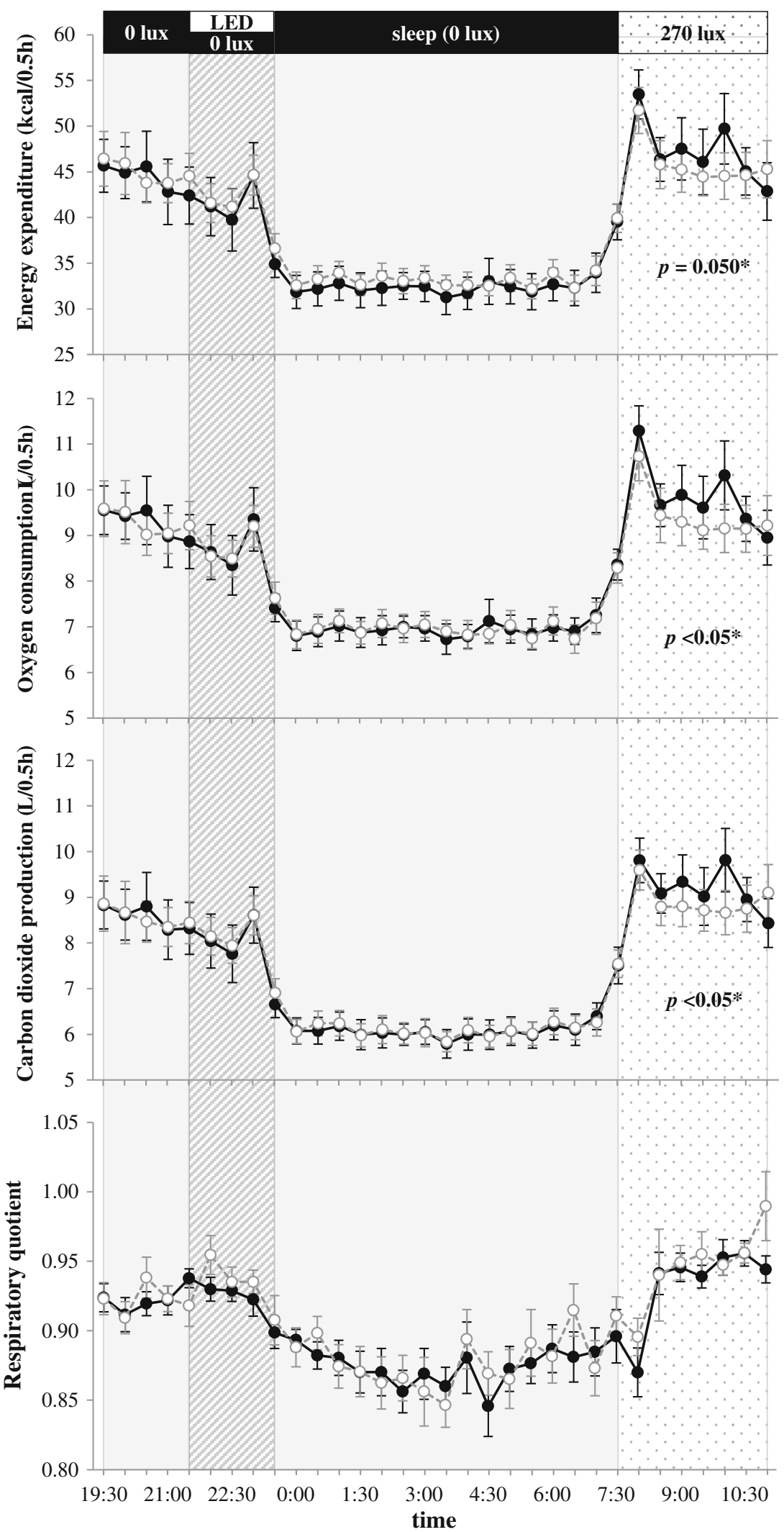


lower for subjects in the blue light condition than those in the no light condition (Fig. 3). In addition, the thermic effect of food after breakfast for subjects in the blue light condition was $57 \pm 7 \mathrm{kcal} / 3 \mathrm{~h}$, corresponding to $7.2 \pm 0.9 \%$ of breakfast, which was significantly lower than the thermic effect for subjects in the no light condition $(74 \pm 7 \mathrm{kcal} / 3 \mathrm{~h}, 9.2 \pm 0.8 \%, p<0.05$, mean $\pm \mathrm{SE})$.

Heart rate and body temperature

Heart rate and body temperature are shown in Table 3. There were no significant differences between two conditions.

\section{Discussion}

Our research showed that exposure to blue LED light suppressed alpha power of EEG in the evening, which is consistent with previous findings that exposure to short wavelength light in the late evening increased alertness [11]. The literature showed that physiological responses such as suppressed melatonin secretion, attenuated decline of body temperature and heart rate in the evening, and changes in sleep architecture were also observed during and after light exposure $[5,11,12,27]$. New findings of the present study were that the effects of blue LED light exposure in the evening were observed the next morning in the front of drowsiness and suppression of energy expenditure.

Continuous polysomnographic monitoring in the present study detected a significant increase in sleep at stages 1 and 2, and a tendency of EEG alpha power to increase the next morning following evening blue light exposure. Alpha waves are generally observed during awake periods with closed eyes, and they are attenuated by opening eyes and mental activity [28].

Reduction in energy expenditure in the morning after evening blue light exposure is due to a reduced wakefulness. Energy expenditure is related to sleep stages and waking after sleep onset is accompanied with an increase in energy expenditure [21]. Likely explanation for drowsiness and suppression of energy expenditure the next morning is a phase shift of the circadian clock by blue light exposure. Sleep is regulated by two mechanisms that work partly independently of each other: the circadian and the homeostatic mechanisms. The circadian clock in the suprachiasmatic nucleus (SCN) is directly connected to photosensitive ganglion cells expressing melanospin via retinohypothalamic tract. The human circadian pacemaker of melatonin secretion is highly sensitive to short wavelength light [5], and Münch et al. [12] observed delayed shift in core body temperature after short wavelength light exposure in the evening and during subsequent sleep. Reduction in energy expenditure and the thermic effect of food the next morning after evening blue light exposure is consistent with the notions that SCN controls circadian energy metabolism, and misalignment between meals and the endogenous circadian cycle increases the risk of obesity [29, 30]. The thermic effect of food is a major form of thermogenesis in humans, and some studies have shown a reduced thermic effect of food in individuals suffering from obesity [31, 32]. Evening blue light exposure, if repeated, may contribute to body weight gain by reducing the thermic effect of food, but it remains to be studied. On the other hand, inadequate sleep, i.e., the role of the homeostatic mechanism as an explanation for subject drowsiness the next morning, was not supported by our observations. Enough time ( $8 \mathrm{~h}$ ) was assigned for time in bed in the present study, and sleep architectures including normal sleep efficiency were similar between the two experimental conditions.

Evening light exposure induces a broad range of physiological responses. Exposure to polychromatic bright light (2,000 lux at 17:00-2:00) in the evening suppresses dietary carbohydrates absorption from the evening meal [33], although absorption of breakfast carbohydrate was not affected [34]. In addition to SCN, areas in the brain responsible for metabolic integration (paraventricular nucleus, dorsomedial hypothalamus and arcuate nucleus) and reward integration (habenula) also receive direct light signals from photosensitive ganglion cells [35]. It is possible that some of physiological responses induced by evening light exposure are induced through a non-SCN pathway.

Contrary to our expectation, sleep architecture and energy metabolism during sleep were not significantly affected by evening exposure to blue light. In previous studies, subjects either received light exposure $(12.1 \mu \mathrm{W} /$ $\mathrm{cm}^{2}$ ) via two glass fiber bundles on custom built goggles $[11,12]$ or gazed at light source $\left(0-42 \mu \mathrm{W} / \mathrm{cm}^{2}\right)$ under the influence of mydriatic agents to dilate pupils [5]. In the present study, subjects in a sitting position did not gaze at the light source $\left(7 \mu \mathrm{W} / \mathrm{cm}^{2}\right)$ set on the ceiling. Therefore, the amount and energy intensity of the light striking the subjects' eyes in the present study seems to be lower than that in the previous studies. In addition, all subjects in the present study were Asian and had brown irises, which limit the effect of light exposure to suppress melatonin secretion compared with subjects with blue eyes [36].

Even relatively low illuminance levels from blue computer screen backgrounds could suppress melatonin secretion and improve subjective and objective measure of alertness [37]. Improvements in alertness and cognitive performance are beneficial in a work environment. 
Although the study shows that evening blue light exposure improves alertness and cognitive function in the evening, findings raise a new concern that evening light exposure has a negative impact on alertness and cognitive function the next morning. One of the limitations of the present study was that the mechanism by which evening light exposure affected the next morning was unclear due to the insufficient evaluation of the subjects' circadian rhythms in this study. It is difficult to generalize the result because of the small number of subjects. Further study that examines the effect of changes in lighting conditions, and exposure timing, alternative variable measures and a different target population would need to be conducted.

Acknowledgments The present study was supported by a Grant-inAid for Scientific Research (No. 23650428). We appreciate the technical support received from Fuji Medical Science (Chiba, Japan).

Conflict of interest The authors have no conflicts of interests or financial ties to disclose.

\section{References}

1. Pimputkar S, Speck JS, DenBaars SP, Nakamura S. Prospects for LED lighting. Nat Photonics. 2009;3:180-2.

2. Brainard GC, Rollag MD, Hanifin JP. Photic regulation of melatonin in humans: ocular and neural signal transduction. J Biol Rhythms. 1997;12:537-46.

3. Zeitzer JM, Dijk DJ, Kronauer R, Brown E, Czeisler C. Sensitivity of the human circadian pacemaker to nocturnal light: melatonin phase resetting and suppression. J Physiol. 2000;526: 695-702.

4. Cajochen C, Zeitzer JM, Czeisler CA, Dijk DJ. Dose-response relationship for light intensity and ocular and electroencephalographic correlates of human alertness. Behav Brain Res. 2000;115:75-83.

5. Brainard GC, Hanifin JP, Greeson JM, Byrne B, Glickman G, Gerner E, et al. Action spectrum for melatonin regulation in humans: evidence for a novel circadian photoreceptor. J Neurosci. 2001;21:6405-12.

6. Thapan K, Arendt J, Skene DJ. An action spectrum for melatonin suppression: evidence for a novel non-rod, non-cone photoreceptor system in humans. J Physiol. 2001;535:261-7.

7. Berson DM, Dunn FA, Takao M. Phototransduction by retinal ganglion cells that set the circadian clock. Science. 2002;295: 1070-3.

8. Panda S, Nayak SK, Campo B, Walker JR, Hogenesch JB, Jegla T. Illumination of the melanopsin signaling pathway. Science. 2005;307:600-4.

9. Qiu X, Kumbalasiri T, Carlson SM, Wong KY, Krishna V, Provencio I, et al. Induction of photosensitivity by heterologous expression of melanopsin. Nature. 2005;433:745-9.

10. Revell VL, Arendt J, Fogg LF, Skene DJ. Alerting effects of light are sensitive to very short wavelengths. Neurosci Lett. 2006;399:96-100.

11. Cajochen C, Münch M, Kobialka S, Kräuchi K, Steiner R, Oelhafen $\mathrm{P}$, et al. High sensitivity of human melatonin, alertness, thermoregulation, and heart rate to short wavelength light. J Clin Endocrinol Metab. 2005;90:1311-6.

12. Münch M, Kobialka S, Steiner R, Oelhafen P, Wirz-Justice A, Cajochen C. Wavelength-dependent effects of evening light exposure on sleep architecture and sleep EEG power density in men. Am J Physiol Regul Integr Comp Physiol. 2006;290:R1421-8.

13. Nicolaidis S. Metabolic mechanism of wakefulness (and hunger) and sleep (and satiety): role of adenosine triphosphate and hypocretin and other peptides. Metabolism. 2006;55:S24-9.

14. Taheri S. The link between short sleep duration and obesity: we should recommend more sleep to prevent obesity. Arch Dis Child. 2006;91:881-4.

15. Centers for Disease Control and Prevention (CDC). Short sleep duration among workers-United States, 2010. MMWR Morb Mortal Wkly Rep. 2012;61:281-5.

16. Chaput JP, Després JP, Bouchard C, Tremblay A. The association between short sleep duration and weight gain is dependent on disinhibited eating behavior in adults. Sleep. 2011;34:1291-7.

17. Lyytikäinen P, Rahkonen O, Lahelma E, Lallukka T. Association of sleep duration with weight and weight gain: a prospective follow-up study. J Sleep Res. 2011;20:298-302.

18. Spiegel K, Leproult R, L'hermite-Balériaux M, Copinschi G, Penev PD, Van Cauter E. Leptin levels are dependent on sleep duration: relationships with sympathovagal balance, carbohydrate regulation, cortisol, and thyrotropin. J Clin Endocrinol Metab. 2004;89:5762-71.

19. Spiegel K, Tasali E, Penev P, Van Cauter E. Brief communication: Sleep curtailment in healthy young men is associated with decreased leptin levels, elevated ghrelin levels, and increased hunger and appetite. Ann Intern Med. 2004;141:846-50.

20. Taheri S, Lin L, Austin D, Young T, Mignot E. Short sleep duration is associated with reduced leptin, elevated ghrelin, and increased body mass index. PLoS Med. 2004;1:e62.

21. Katayose Y, Tasaki M, Ogata H, Nakata Y, Tokuyama K, Satoh M. Metabolic rate and fuel utilization during sleep assessed by whole-body indirect calorimetry. Metabolism. 2009;58:920-6.

22. Ministry of Health, Labour and Welfare of Japan. The National Health and Nutrition Survey 2010 [in Japanese]. 2012. Available from: http://www.mhlw.go.jp/bunya/kenkou/eiyou/d1/h22-hou koku-01.pdf.

23. Rechtschaffen A, Kales A. A manual of standardized terminology, techniques and scoring system for sleep stages of human subjects. Washington DC: US Government Printing Office; 1968.

24. Tokuyama K, Ogata H, Katayose Y, Satoh M. Algorithm for transient response of whole body indirect calorimeter: deconvolution with a regularization parameter. J Appl Physiol. 2009;106:640-50.

25. Zhang L, Samet J, Caffo B, Bankman I, Punjabi NM. Power spectral analysis of EEG activity during sleep in cigarette smokers. Chest. 2008;133:427-32.

26. Westerterp KR. Diet induced thermogenesis. Nutr Metab (Lond). 2004; $1: 5$

27. Lockley SW, Brainard GC, Czeisler CA. High sensitivity of the human circadian melatonin rhythm to resetting by short wavelength light. J Clin Endocrinol Metab. 2003;88:4502-5.

28. Curcio G, Casagrande M, Bertini M. Sleepiness: evaluating and quantifying methods. Int J Psychophysiol. 2001;41:251-63.

29. Coomans CP, van den Berg SA, Lucassen EA, Houben T, Pronk AC, van der Spek RD, et al. The suprachiasmatic nucleus controls circadian energy metabolism and hepatic insulin sensitivity. Diabetes. 2013;62:1102-8.

30. Morikawa Y, Nakagawa H, Miura K, Soyama Y, Ishizaki M, Kido $\mathrm{T}$, et al. Effect of shift work on body mass index and metabolic parameters. Scand J Work Environ Health. 2007;33:45-50.

31. Segal KR, Edaño A, Blando L, Pi-Sunyer FX. Comparison of thermic effects of constant and relative caloric loads in lean and obese men. Am J Clin Nutr. 1990;51:14-21.

32. Thörne A, Näslund I, Wahren J. Meal-induced thermogenesis in previously obese patients. Clin Physiol. 1990;10:99-109. 
33. Hirota N, Sone Y, Tokura H. Effect of evening exposure to dim or bright light on the digestion of carbohydrate in the supper meal. Chronobiol Int. 2003;20:853-62.

34. Hirota N, Sone Y, Tokura H. Effect of evening exposure to bright or dim light after daytime bright light on absorption of dietary carbohydrates the following morning. J Physiol Anthropol. 2010;29:79-83.

35. Albrecht U. Timing to perfection: the biology of central and peripheral circadian clocks. Neuron. 2012;74:246-60.
36. Higuchi S, Motohashi Y, Ishibashi K, Maeda T. Influence of eye colors of Caucasians and Asians on suppression of melatonin secretion by light. Am J Physiol Regul Integr Comp Physiol. 2007;292:R2352-6.

37. Cajochen C, Frey S, Anders D, Späti J, Bues M, Pross A, et al. Evening exposure to a light-emitting diodes (LED)-backlit computer screen affects circadian physiology and cognitive performance. J Appl Physiol. 2011;110:1432-8. 